\title{
Modulation Ranges of Different Sensations for Coding Electrically Evoked Tactile Sensory Feedback
}

This paper was downloaded from TechRxiv (https://www.techrxiv.org).

\section{LICENSE}

CC BY 4.0

SUBMISSION DATE / POSTED DATE

28-05-2021 / 30-05-2021

\section{CITATION}

Zhang, Jie; Hao, Manzhao; Yang, Fei; Liang, Wenyuan; Bi, Sheng; Chou, Chih-hong; et al. (2021): Modulation Ranges of Different Sensations for Coding Electrically Evoked Tactile Sensory Feedback. TechRxiv. Preprint. https://doi.org/10.36227/techrxiv.14693934.v1

\section{$\mathrm{DOI}$}

10.36227/techrxiv.14693934.v1 


\title{
Modulation Ranges of Different Sensations for Coding Electrically Evoked Tactile Sensory Feedback
}

\author{
Jie Zhang, Manzhao Hao, Fei Yang, Wenyuan Liang, Sheng Bi, Chih-hong Chou, and Ning Lan
}

\begin{abstract}
Objective: The ability to perceive prosthetic grasping may enable amputees to better interact with external objects. This may require customized coding of multiple sensory feedback for each amputee. This study was to develop a protocol to determine optimal modulation ranges of sensations elicited by transcutaneous electrical nerve stimulation (TENS) in amputees with evoked tactile sensation (ETS). Methods: 5 forearm amputees and 10 ablebodied subjects participated in this study. Surface electrodes were placed at the most sensitive point of projected finger map at the stump of amputees. Effects of stimulus frequency on sensory types were assessed, and the amplitude/pulse-width perceptual threshold relationship for each sensation was characterized. Modulation ranges of pulse width at fixed amplitude were determined for optimal coding of sensory feedback. Sensitivity of vibration and buzz sensations was measured using just noticeable difference (JND). A linear sensory coding was implemented in amputees in finger-specific identification experiments. Results: Vibration and buzz sensations were consistently evoked at $20 \mathrm{~Hz}$ and $50 \mathrm{~Hz}$ respectively. The perceptual thresholds of different sensations displayed an inverse relation between current amplitude and pulse width. The JND of vibration and buzz was independent of reference pulse width at $12.84 \pm 7.23$ $\mu s$ and $15.21 \pm 6.47 \mu$ s in able-bodied subjects, and 14.91 $\pm 10.54 \mu \mathrm{s}$ and $11.30 \pm 3.42 \mu \mathrm{s}$ in amputees. The overall accuracy of finger-specific identification was $77.85 \%$ in amputees. Conclusion: This study developed an effective protocol to evaluate the modulation ranges of sensations evoked by TENS for finger-specific perception in amputees. Significance: Personalized sensory coding facilitates customization of ETS-based sensory feedback to amputees.
\end{abstract}

Index Terms: Sensory feedback, evoked tactile sensation, hand prosthesis, modulation of sensory perception, transcutaneous electrical nerve stimulation

This research was supported in part by a grant from the National Key R\&D Program of China (No. 2017YFA0701104), a grant from the Natural Science Foundation of China (No. 81630050), a grant from Key Areas R\&D Program of GuangDong Province (No. 2020B0909020004), and a grant from Science and Technology Commission of Shanghai Municipality (No. 20DZ2220400). (Corresponding authors: Ning Lan (email: ninglan@sjtu.edu.cn); Manzhao Hao (email: haomzh@sjtu.edu.cn)

J. Zhang and F. Yang are with the Laboratory of NeuroRehabilitation Engineering, School of Biomedical Engineering, Shanghai Jiao Tong University, Shanghai, China.

M. Hao, C. Chou and N. Lan are with the Laboratory of NeuroRehabilitation Engineering, School of Biomedical Engineering, and Institute of Medical Robotics, Shanghai Jiao Tong University, Shanghai, China.

W. Liang and $\mathrm{S}$. Bi are with the National Research Center for Rehabilitation and Technical Aids, Beijing, China.

\section{INTRODUCTION}

I oss of hand devastates motor and sensory functions in forearm amputees. Providing sensory feedback to amputees, particularly haptic awareness during prosthetic grasping, is critical to improve the performance of hand prosthesis [1]. A variety of technologies have been developed to reconstruct sensory feedback based on invasive and noninvasive neural interfaces [2]-[7] The effect of restored sensory functions depends on the richness and naturalness of sensory information conveyed via these techniques. Sensation qualities, such as referred hand percepts, sensation type, and perceived intensity are major ways for conveying sensory information to amputees. The relationship between sensations and stimulation parameters is pivotal to allow coding external information to sensory stimulation.

Somatotopic sensory information of hand could be encoded by directly electrically stimulating the sensory neurons in brain [2], [8], [9] or peripheral nerves [10]-[12], and non-invasively mechanically or electrically stimulating the projected finger map (PFM) area of the stump skin [6], [13], [14]. The interface, the implanted location, and current parameters used in invasive techniques could affect the perceptual qualities [9], [10], [15] and multiple sensory modalities [8], [16]-[18]. While studies of peripheral nerve electrical stimulation show the sophisticated sensory function [17], [19], [20] with a relatively long time [11], [12], [21], non-invasive sensory feedback studies are still relatively limited in the use of sensation qualities [22], [23].

In previous studies, we assessed TENS as a non-invasive way to elicit finger-specific sensations in PFM, namely evoked tactile sensation (ETS) [23], [24]. A variety of sensations, such as touch, pressure, buzz, vibration, numb and tingling, could be evoked, which may result from activation of different sensory nerve fibers of cutaneous receptors [25]-[27]. This provides a possible means to encode rich sensory information. Sensations evoked by TENS could be dependent on stimulus parameters [27]-[30], electrode size [25], [31], electrode material [25], and skin location [28], [32]. Sensation type, perceptual threshold, and intensities could be modulated by amplitude, pulse width, or frequency of stimulus [24], [33], [34]. Frequency modulation was found easier to perceive and interpret than intensity modulation, the latter was varied by modulation of amplitude and pulse width [29], [30]. However, the level of perceived intensity with amplitude and pulse width modulations was finer than that of frequency modulation [32]. Furthermore, in the restricted PFM areas at the stump, smaller electrodes must be used for multi-site stimulation, which may inevitably lose some ability to recruit different types of sensory fibers [31]. Therefore, how to optimally encode sensory information with 
limited types of sensations is a critical question when fitting the ETS-based feedback to an amputee.

In this study, we evaluated sensory qualities evoked by TENS on the normal skin of able-bodied subjects and PFM of amputees using small electrodes. The pulse width of the stimulus was modulated here for its wide modulation range [24] and fine regulation of perceptual intensity [32]. We measured the perceptual threshold of attainable sensations at different frequencies, amplitudes, and pulse width to determine the relationship among these parameters. The sensitivity for vibration and buzz was assessed by quantifying just noticeable difference (JND) in pulse width in both able-bodied and amputee subjects. A coding strategy with fixed amplitude and pulse width modulation was tested in amputees in fingerspecific perception experiment, demonstrating the feasibility of optimal coding of sensory information with ETS-based, noninvasive feedback for amputees. Part of preliminary results was presented in a conference proceeding [35].

\section{Methods}

\section{A. Subjects}

5 amputee subjects (S1-S5, all males, $62 \pm 5$ years old) (see Table 1) and 14 able-bodied subjects (H1-H14, 25 \pm 2 years old, 9 females and 5 males) were recruited in this study. All subjects had normal cognitive function. They were informed about the experimental procedures and signed the consent form before the experiments. This study was approved by the Institutional Review Board for Human Research Protections, Shanghai Jiao Tong University. S1, S3, S4, and S5 participated in all experiments except Exp. 1A. Due to physical reasons, S2 only carried out the Exp. 3 and dropped out of this study. Four ablebodied subjects (H1-H4) took part in all the experiments except Exp. 3. H6-H8 participated in Exp. 1A and Exp. 2. H5 and the other able-bodied subjects (H12-H14) only took part in Exp. 1B and Exp. 2, respectively.

\section{B. Experimental setup}

In this study, a multi-channel, programmable sensory feedback system (Fig. 1(a)) was developed to deliver electrical stimulation and achieve real-time pressure feedback from the prosthetic hand to subjects [23]. The current stimulus was a biphasic, charge-balanced, cathode-first pulse train with $10 \mu \mathrm{s}$ inter-pulse interval. When using program-I of the system, the output channel and the parameters of amplitude, frequency, and pulse width could be set by the host computer. In program-II, the system would collect pressure signals through the force sensors (FlexiForce A201, Tekscan Inc, the United States) mounted at the fingertips of the prosthetic hand, then convert pressure signals into multi-channel electrical stimuli in realtime. A trigger signal from a press bar by the subject was sampled by the system for event detection.

To define the projected finger map (PFM) on the stump of forearm amputees with evoked tactile sensation (ETS), we used a metal pen with a round tip of $2 \mathrm{~mm}$ in diameter to press the stump skin, and marked the most sensitive point (MSP) of each finger with a cross according to the perception of amputees. The PFM distribution located at the stump was personalized for each amputee subject (Fig. 1(b)). All of those five amputee subjects
Table 1 General information for amputee subjects

\begin{tabular}{cccc}
\hline $\begin{array}{c}\text { Subjects } \\
\text { (Gender, age })\end{array}$ & $\begin{array}{c}\text { Amputation } \\
\text { side }\end{array}$ & $\begin{array}{c}\text { Amputation cause } \\
\text { and time (years) }\end{array}$ & $\begin{array}{c}\text { Level of } \\
\text { amputation }\end{array}$ \\
\hline S1 (M,67) & Left, & Trauma, 44 & Below elbow \\
S2 $(\mathrm{M}, 56)$ & Left & Trauma, 4 & At wrist \\
S3 $(\mathrm{M}, 56)$ & Right & Trauma, 9 & Below elbow \\
S4 $(\mathrm{M}, 67)$ & Left & Trauma, 14 & At wrist \\
S5 $(\mathrm{M}, 63)$ & Right & Trauma, 35 & At wrist \\
\hline
\end{tabular}

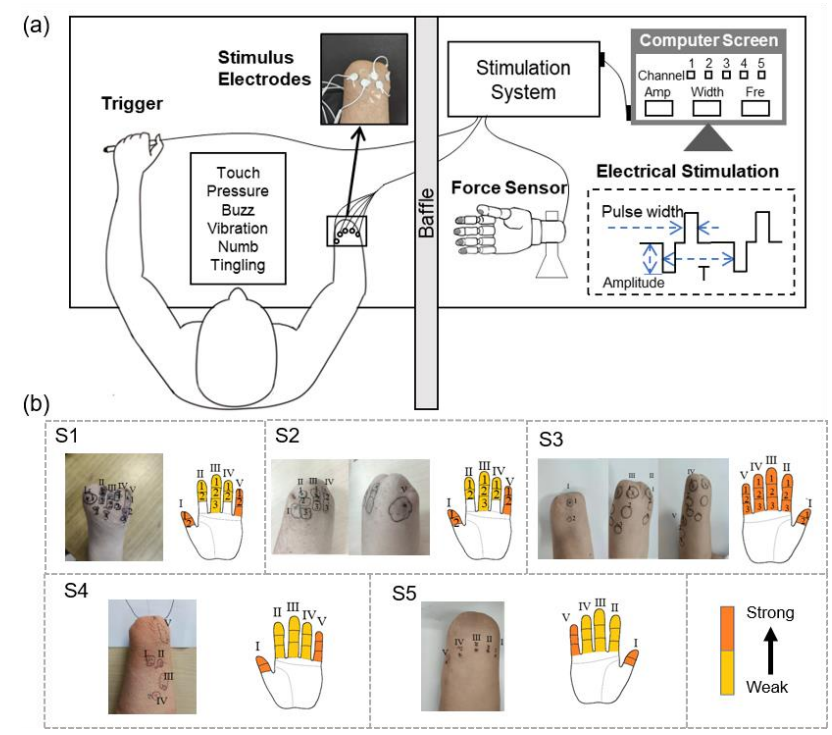

Fig. 1. (a) Experiment setup and the illustration of current stimulus. $\mathrm{T}=$ 1/frequency. (b) Identified projected finger maps (PFMs) of S1, S2, S3, S4, and S5. The PFM regions of thumb (I), index (II), medius (III), ring (IV), and pinky (V) were marked with circles and the most sensitive point (MSP) of the PFM region for each finger was labeled by a cross. Numbers $(1,2,3)$ indicate finger phalanges.

could sense all the five fingers with strong or weak sensations. S1, S2 and S3 could identify feeling at some individual phalanges. All PFM regions had areas of relative size for all amputee subjects expect S5, who had discrete sensitive points.

The MSP of each finger was determined as the stimulation site for amputee subjects. For able-bodied subjects, one stimulation site was located on the volar side, $1 / 3$ of the way from the left forearm to the wrist. The electrical stimulation was delivered by the circular nonwoven electrode of $10 \mathrm{~mm}$ in diameter (Yancheng Dalun Medical Equipment Co. Ltd, China), and return electrode $(25 \mathrm{~mm}$ in diameter) was placed near the elbow of the ipsilateral arm.

\section{Exp. 1A: Pulse-width perceptual threshold of sensa-tions at different frequencies}

To assess the effect of current frequency, the pulse-width perceptual threshold of sensations was evaluated with one fixed current amplitude at four different frequencies, which were 10 $\mathrm{Hz}, 20 \mathrm{~Hz}, 50 \mathrm{~Hz}$, and $100 \mathrm{~Hz}$. At first, the pulse width was set as $200 \mu \mathrm{s}$ at the frequency of $20 \mathrm{~Hz}$, the fixed current amplitude of each site to be evaluated was set as the first perceptual value, starting at $1 \mathrm{~mA}$ in increments of $0.5 \mathrm{~mA}$. Each stimulus lasted 
for 5 seconds.

For each stimulation site, there were 4 evaluation blocks, with each block tested at one frequency. The pulse width increased from $20 \mu$ s with a step of $20 \sim 40 \mu$ s with the fixed amplitude and frequency. Each stimulation lasted for 5 seconds. The subjects were asked to choose the matched sensation from six sensations (touch, pressure, buzz, vibration, numb, and tingling) characterized in the previous study [24]. If they reported tingling sensation or pulse width increased to $600 \mathrm{us,}$ this block ended. The stimulus parameters and the matched sensations were recorded. The pulse-width perceptual threshold was defined as the pulse width value that each sensation first appeared. 10-15 minutes break between two blocks was given to the subject to avoid the perceptual adaptation.

\section{Exp. 1B: Relationship of current amplitude and pulse-width perceptual threshold for sensations}

The pulse-width perceptual thresholds of sensations at $20 \mathrm{~Hz}$ and $50 \mathrm{~Hz}$ were obtained with the current amplitude ranging from $1 \mathrm{~mA}$ to $7 \mathrm{~mA}$ with an interval of $0.5 \mathrm{~mA}$. Each block was tested at one fixed amplitude. Thus there were 13 blocks at 20 $\mathrm{Hz}$ or $50 \mathrm{~Hz}$. In each block, the perceptual thresholds of sensations were measured with a similar process as in Exp. 1A. Each stimulation lasted for 3 seconds.

For each sensation that occurred in more than two blocks of a single evaluated site at the same frequency, the relation between pulse-width perceptual threshold and current amplitude at $20 \mathrm{~Hz}$ or $50 \mathrm{~Hz}$ was fitted by the following function.

$$
L T_{S}=c /(A-b)
$$

where $A$ is the amplitude, $b$ and $c$ are the constant parameters of the fitted curve, $L T$ is the pulse-width perceptual threshold of the sensation $S$ at amplitude $A$.

The modulation area of the sensation was defined as the region between the fitted curve of this sensation and the next sensation that appeared with the increase of pulse width, which was calculated as follows,

$$
R=\int_{A_{\min }}^{A_{\max }}\left(L T_{S_{1}}(A)-L T_{S_{0}}(A)\right) d A
$$

where $R$ is the modulation area of the sensation $S_{0}, L T_{S_{0}}(A)$ and $L T_{S_{1}}(A)$ are the pulse-width perceptual threshold of the sensation $S_{0}$ and the next sensation $S_{1}$ at fixed amplitude $A$, respectively. If there was no next sensation, $L T_{S_{1}}(A)=$ $600 \mu \mathrm{s} . A_{\min }$ and $A_{\max }$ are the minimum and maximum amplitude that $S_{0}$ appeared respectively.

\section{E. Exp. 1C: Pulse-width modulation ranges of sensations}

For each evaluated site, the same measurement as Exp. 1A of the pulse-width perceptual threshold was performed three times with each stimulus lasted for 3 seconds. The choice of fixed amplitude referred to the result of Exp.1B to achieve the wider modulation range.

The upper threshold of the sensation was defined as the pulse width value when this sensation disappeared. The mean value of three differences between the perceptual threshold and upper threshold obtained from three times tests was calculated as the modulation range of the sensation. The primary sensation was defined as the sensation that appeared in more than half of the able-bodied subjects or amputee subjects, separately. Then, the averaged modulation ranges of primary sensations across group subjects were further calculated.

\section{F. Exp. 2: Sensitivity for vibration and buzz sensation}

Since vibration and buzz were primary sensations at $20 \mathrm{~Hz}$ and $50 \mathrm{~Hz}$ respectively, we assessed their sensitivities in this experiment. Sensitivity was characterized by the just noticeable difference (JND) which was defined as the minimum amount of change in pulse width that produced a noticeable difference.

The reference stimulus and comparison stimulus were delivered in sequence with a 2 -sec inter-stimulus interval. Each stimulation lasted for 3 seconds. The initial pulse width of the comparison stimulus was 5 us higher than that of the reference stimulus. The reference stimulus remained unchanged and the pulse width of the comparison stimulus was increased by a step of $5 \mu$ s until the subject could identify their difference of intensity. The pulse width difference between the stimuli pair that produced a noticeable difference was recorded as JND.

The reference stimulus increased from the perceptual threshold to the upper threshold of vibration/buzz sensation by a step of 50 us. The modulation range of vibration/buzz sensation was obtained in Exp. 1C. S3 was unable to make a clear identification, so we did not collect complete data of sensitivity from this subject.

The measurement of JND was repeated three times at each reference stimulus. The mean value was calculated as JND at this reference stimulus. The JND of vibration and buzz sensation across all reference stimuli was calculated.

\section{G. Exp. 3: The finger-specific identification}

The buzz sensation at $50 \mathrm{~Hz}$ was chosen to encode the fingertip pressure of the prosthetic hand. The fixed amplitude and modulation range of buzz sensation was measured in Exp. $1 \mathrm{C}$. The pulse width was linearly proportional to the collected force as follows.

$$
\begin{array}{lc}
W=0, & P<P_{\text {min }} \\
W=\frac{W_{\max }-W_{\min }}{P_{\max }-P_{\min }} *\left(P-P_{\min }\right)+W_{\min }, & P_{\min } \leq P<P_{\max } \\
W=W_{\max } & P \geq P_{\max }
\end{array}
$$

where $W$ is the output pulse width, $P$ is the pressure collected by force sensors, $P_{\max }$ is the maximum pressure set to $15 \mathrm{~N}$, and $P_{\min }$ is the minimum pressure set to $1.5 \mathrm{~N}$. $W_{\min }$ and $W_{\max }$ were the perceptual threshold and upper threshold of buzz sensation in pulse width, respectively.

Before the test, five fingers of the prosthetic hand were pressed three times to familiarize the subject with the experiment setup (Fig. 1(a)). During the test, a paper baffle was used to block the participant's view of the prosthetic hand. The experimenter pressed force sensors in random force and order. The subject was asked to report the perceived finger and press the trigger bar simultaneously. Each finger was randomly pressed 24 times over 6 blocks. The pressure, trigger signal, and identification were recorded.

The accuracy of finger-specific identification was calculated. Reaction time was the time interval between the moments of force sensor contact and trigger pressing by the subject. 


\section{H. Statistical analysis}

All data were analyzed using MATLAB (R2020a, The MathWorks, Natick, US). They were reported as mean \pm standard deviation. The Mann-Whitney U-test was used to detect whether there was a significant difference in the modulation range or sensitivity between different sensations or subjects at the 5\% significance level. The reaction time between correct identification and incorrect identification was also analyzed by this test.

\section{Results}

\section{A. Perceptual threshold for sensations at different frequencies}

We evaluated the perceptual threshold of sensations with pulse width of TENS for four frequencies at fixed amplitude in 10 able-bodied subjects (Fig. 2). The types and perceptual thresholds of sensations varied with frequencies. Four types of sensations were perceived at $10 \mathrm{~Hz}$ and $100 \mathrm{~Hz}$, while five sensations could be evoked at $20 \mathrm{~Hz}$ and $50 \mathrm{~Hz}$. These sensations were perceived in the order of touch, buzz, vibration, numb and tingling. The pulse-width perceptual threshold of these sensations increased gradually at each fixed frequency. Touch and tingling sensations could be evoked at all four frequencies. Vibration sensation was elicited in most subjects perceived in most subjects at $50 \mathrm{~Hz}$ and $100 \mathrm{~Hz}$. It was evident

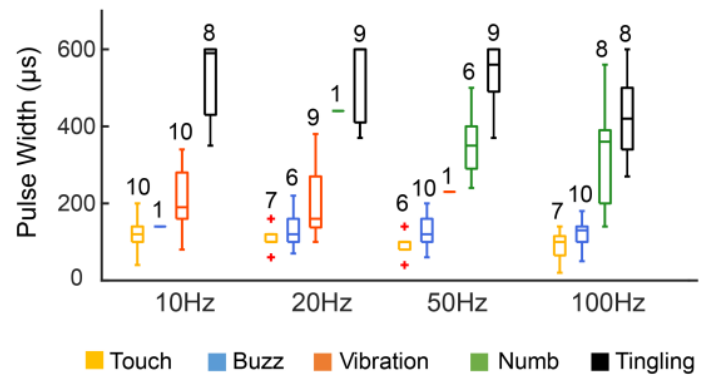

Fig. 2. Pulse width perceptual threshold for sensations of the able-bodied subjects $(\mathrm{N}=10)$ at $10 \mathrm{~Hz}, 20 \mathrm{~Hz}, 50 \mathrm{~Hz}$, and $100 \mathrm{~Hz}$. The perceptual thresholds of sensations are expressed in different colors. The number at the top of each boxplot represents the number of subjects with this sensation.

that more sensation types were elicited with $20 \mathrm{~Hz}$ and $50 \mathrm{~Hz}$ stimulation.

\section{B. Modulation ranges of sensations at different amplitudes}

Perceptual thresholds of pulse width at $20 \mathrm{~Hz}$ and $50 \mathrm{~Hz}$ frequencies were further evaluated with pulse width modulation at different amplitudes in five able-bodied subjects and four amputees (Fig. $3 \&$ Fig. S1). The pulse width-amplitude data at $20 \mathrm{~Hz}$ and $50 \mathrm{~Hz}$ were fitted to an inverse function (Eq. (1)) in able-bodied subjects and amputee subjects (Table S1-S5). The (a)

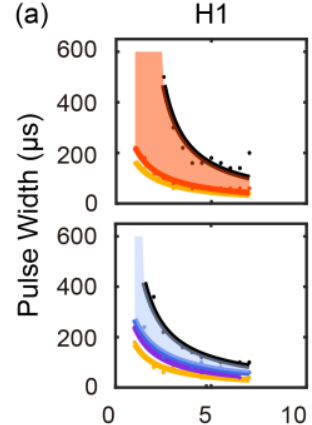

(c)

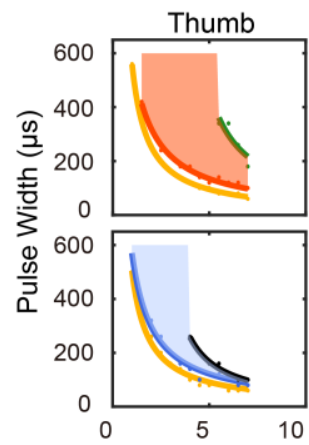

$\mathrm{H} 2$
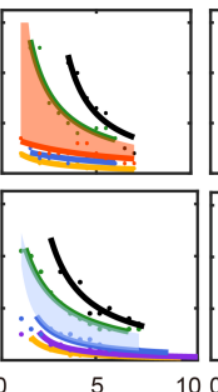

H3
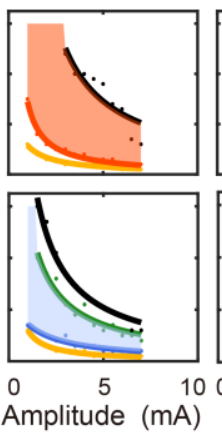

S1

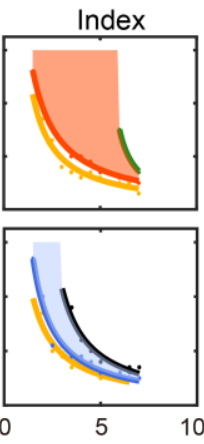

Medius
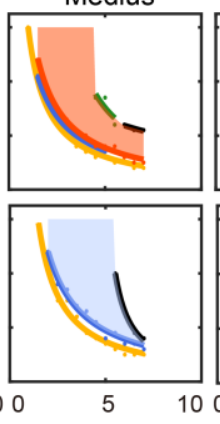

Amplitude $(\mathrm{mA})$

Touch
$\mathrm{H} 4$
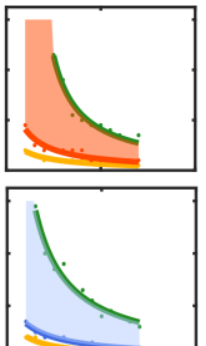
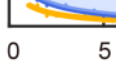

$5 \quad 10$
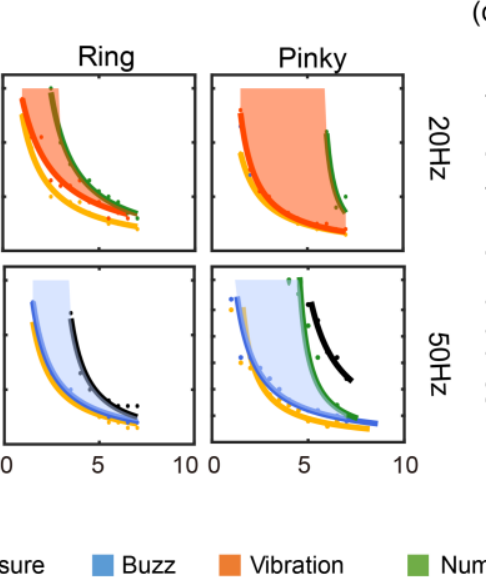

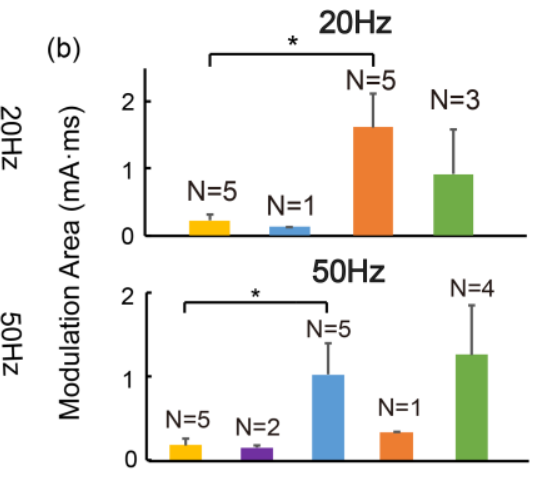

(d)
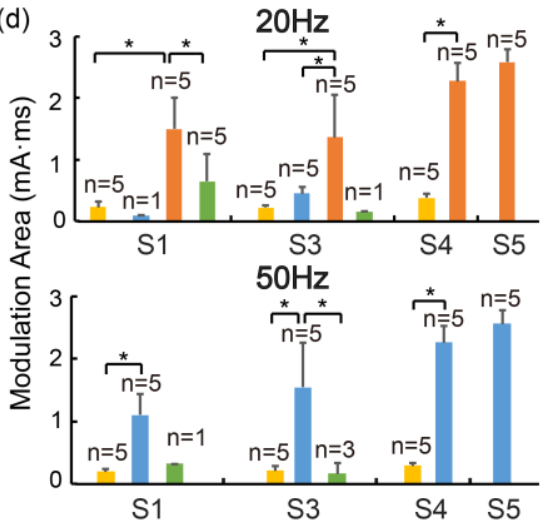

Numb

Tingling

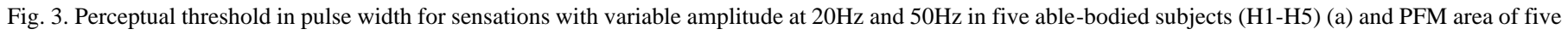

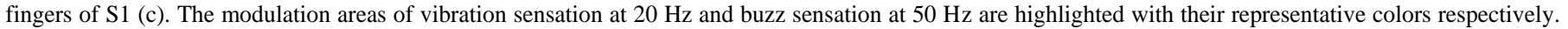

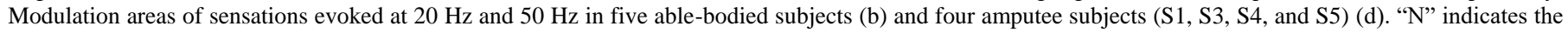

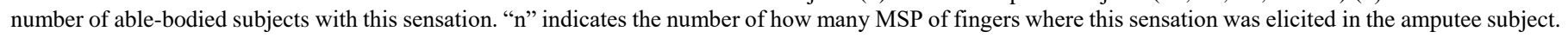
Statistical significance is indicated with '*' $(p<0.05)$. 
perceptual thresholds in pulse width at all sensory types varied reciprocally with amplitude in both able-bodied subjects and amputee subjects (Fig. 3(a), 3(b) \& Fig. S1). With increased pulse width, the occurrence of sensations usually followed the order of touch, vibration, and buzz, numb and tingling. The modulation areas of sensations were presented in Fig. 3(b) and 3(d). We found that the vibration and buzz sensations were predominantly present in all five able-bodied subjects and four amputee subjects at $20 \mathrm{~Hz}$ and $50 \mathrm{~Hz}$ (Fig. 3(b) \& 3(d)). Vibration displayed the largest modulation area at $20 \mathrm{~Hz}$, while the largest modulation area of buzz sensation appeared at $50 \mathrm{~Hz}$ in both able-bodied and amputee subjects. Touch sensation could be felt by five able-bodied subjects and three amputee subjects (S1, S3, and S4). The modulation areas of vibration and buzz sensations were significantly greater than that of touch sensation in able-bodied subjects and three amputee subjects ( $\mathrm{S} 1, \mathrm{~S} 3$, and $\mathrm{S} 4)$ at $20 \mathrm{~Hz}$ and $50 \mathrm{~Hz}$ respectively $(\mathrm{p}<0.05)$. The modulation area of numb sensation was significantly smaller than that of vibration in S1 and that of buzz sensation in S3 at $20 \mathrm{~Hz}$ and $50 \mathrm{~Hz}$ respectively $(\mathrm{p}<0.05)$. The modulation area of buzz was significantly smaller than that of vibration in S3 at $20 \mathrm{~Hz}(\mathrm{p}<0.05)$. One amputee subject (S5) was able to perceive only vibration at $20 \mathrm{~Hz}$, or buzz at $50 \mathrm{~Hz}$ (Fig. 3(d) \& Fig. S1).

\section{Modulation ranges for different sensations at the fixed amplitude}

In the following experiments, we further examined the ranges of pulse width modulation of different sensations at $20 \mathrm{~Hz}$ and $50 \mathrm{~Hz}$ with a fixed amplitude, which was optimally chosen for a wide modulation range of pulse width. Results were presented in Fig. 4 for able-bodied subjects and in Fig. 5 for amputee subjects, respectively. It was clear that vibration and buzz were the predominantly perceived sensations by both able-bodied and amputee subjects at $20 \mathrm{~Hz}$ and $50 \mathrm{~Hz}$, respectively, which was consistent with previous results. The average range of pulse width modulation for vibration was widest among all sensations in both groups of subjects at $20 \mathrm{~Hz}$ (Fig. 4(a) \& Fig. 5(a)). At $50 \mathrm{~Hz}$, the average range of pulse width modulation for buzz was the widest among all subjects (Fig. 4(b) \& Fig. 5(b)). Touch sensation was evoked at $20 \mathrm{~Hz}$ and $50 \mathrm{~Hz}$ in most subjects. The average modulation range of vibration and buzz sensation was significantly higher than that of other primary sensations in both groups of subjects at $20 \mathrm{~Hz}$ and $50 \mathrm{~Hz}$, respectively $(\mathrm{p}<0.05)$ (Fig. 4(c) \& Fig. 5(c)). Only S3 and S4 perceived tingling sensation at this simulation amplitude. Fixing stimulation amplitude may allow a rapid determination of optimal ranges of pulse width modulation for each amputee when fitting ETSbased sensory feedback.

\section{Sensitivity for vibration and buzz sensation}

Pulse width sensitivity of vibration and buzz sensations were examined in able-bodied subjects $(\mathrm{N}=10)$ and amputee subjects (S1, S4, and S5) (Fig. 6 \& Fig. S2), which was measured by just noticeable differences (JND). In able-bodied subjects $(\mathrm{N}=10)$, the JNDs of vibration and buzz sensations were not dependent on reference pulse width (Fig. 6(a)). Similar independence of JND with reference pulse width was observed in amputee subjects (Fig. 6(b) \& Fig. S2).

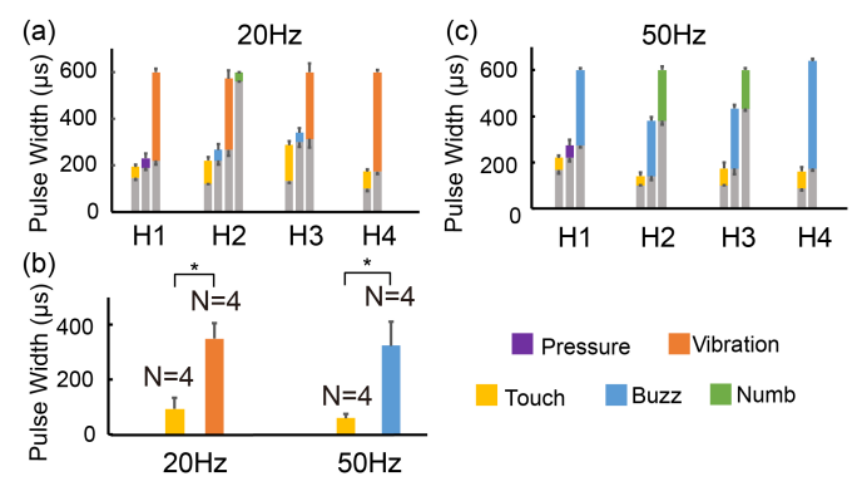

Fig. 4. Modulation ranges of pulse width for sensations with fixed amplitude at $20 \mathrm{~Hz}$ (a) and $50 \mathrm{~Hz}$ (b) in four able-bodied subjects. The colored bars indicate modulation ranges of pulse width for sensations (Mean + SD). Gray bar presents the perceptual threshold of the sensation (Mean - SD). (c) displays the modulation range for primary sensations at $20 \mathrm{~Hz}$ and $50 \mathrm{~Hz}$ in four ablebodied subjects. 'N' represents the number of able-bodied subjects with this sensation. Statistical significance is indicated with '*' $(p<0.05)$.

(a)
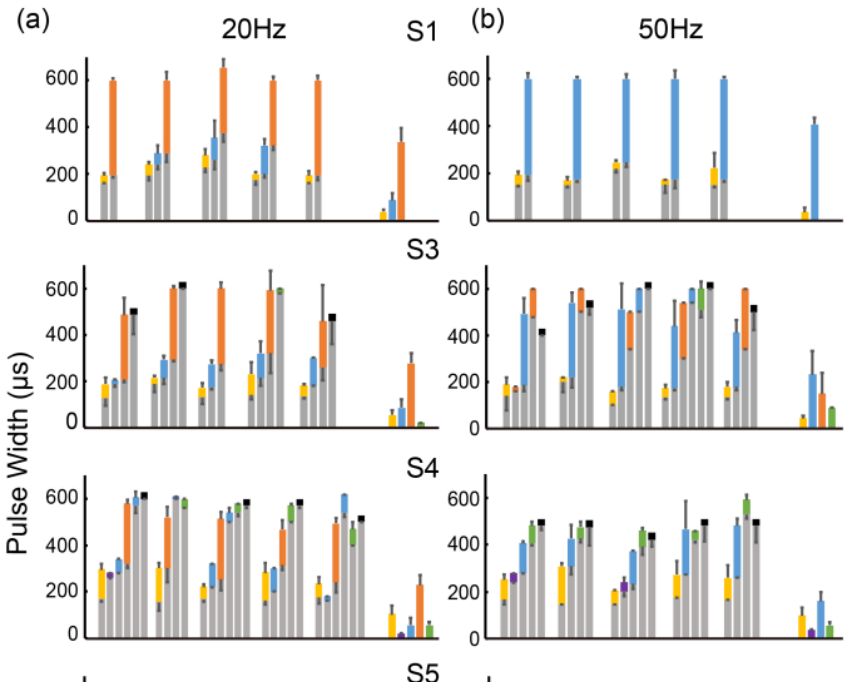

$\$ 4$
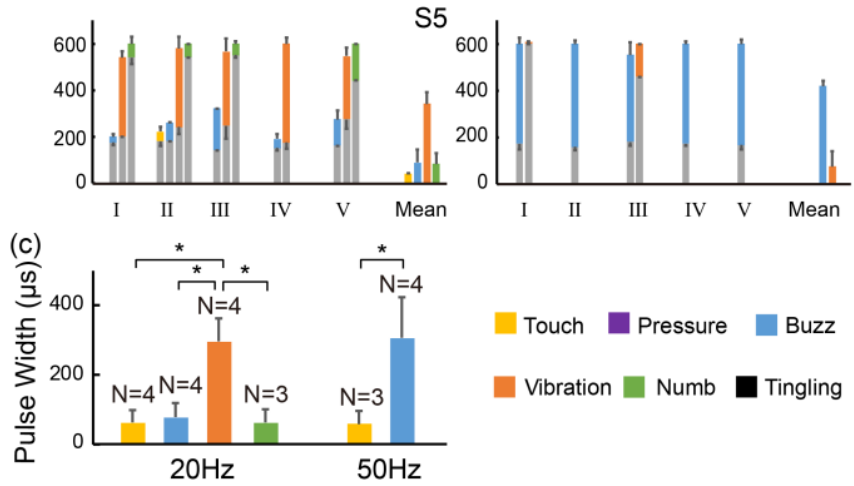

Fig. 5. Modulation ranges of pulse width for sensations with fixed amplitude at $20 \mathrm{~Hz}$ (a) and $50 \mathrm{~Hz}$ (b) on PFM regions of thumb (I), index (II), medius (III), ring (IV), and pinky (V) for four amputee subjects. The color bar and gray bar are represented in the same way as Fig. 4. The black bar with the fixed range of $30 \mu \mathrm{s}$ is used to present the appearance of tingling sensation. The last column shows the modulation range (mean $\pm \mathrm{SD}$ ) for sensations on all five PFMs. (c) displays the pulse width modulation range for primary sensations in four amputees at $20 \mathrm{~Hz}$ and $50 \mathrm{~Hz}$. 'N' represents the number of amputee subjects with this sensation. Statistical significance is indicated with '*' $(p<0.05)$. 


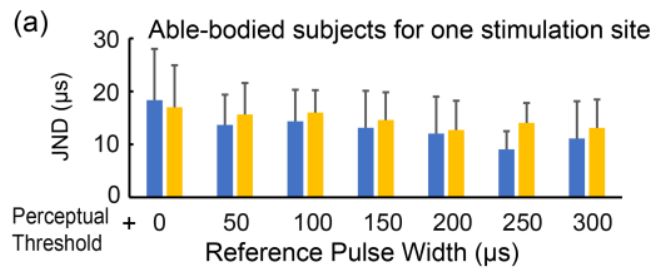

(b) Amputee subjects for five stimulation sites

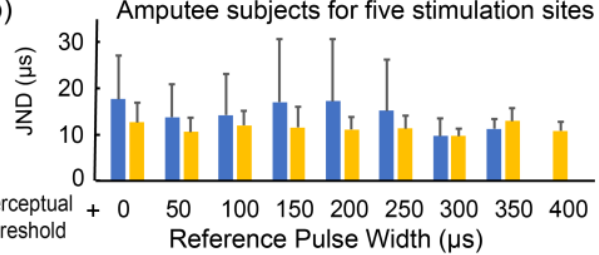

(c)

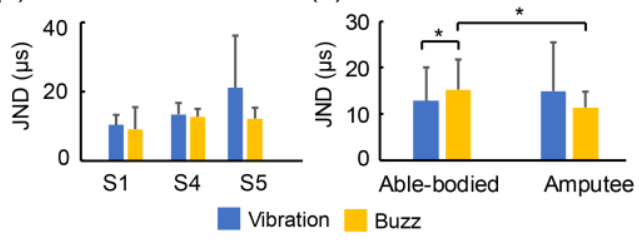

Fig. 6. Sensitivity of vibration and buzz sensation at different pulse width reference of stimulus in able-bodied subjects $(\mathrm{N}=10)$ for one stimulation site (a) and three amputee subjects (S1, S4, and S5) for five PFMs (b). Sensitivity was characterized by just noticeable differences (JND). (c) depicts the JND of vibration and buzz sensation in S1, S4 and S5. (d) presents the JND of vibration and buzz for able-bodied subjects $(\mathrm{N}=10)$ and amputee subjects $(\mathrm{S} 1, \mathrm{~S} 4$, and S5). Statistical significance is indicated with ' $*$ ' $(p<0.05)$.

For three amputee subjects (S1, S4, and S5), the mean JNDs of vibration and buzz sensation were shown in Fig. 7(c), which were $10.34 \pm 2.84 \mu \mathrm{s}, 13.33 \pm 3.3 \mu \mathrm{s}$, and $21.05 \pm 15.02 \mu \mathrm{s}$ for vibration, and $9.08 \pm 2.34 \mu \mathrm{s}, 12.64 \pm 3.11 \mu \mathrm{s}$, and 12.17 $\pm 3.48 \mu \mathrm{s}$ for buzz, respectively. There was no significant difference in the sensitivity of vibration and buzz for S1, S4, and S5 ( $\mathrm{p}>0.05)$.

The JNDs of vibration and buzz sensation in able-bodied subjects $(\mathrm{N}=10)$ and amputee subjects ( $\mathrm{S} 1, \mathrm{~S} 4$, and $\mathrm{S} 5)$ were displayed in Fig. 6(d). The average JNDs for vibration and buzz sensation was $12.84 \pm 7.23 \mu$ s and $15.21 \pm 6.47 \mu$ s for ablebodied subjects, and $14.91 \pm 10.54 \mu$ s and $11.30 \pm 3.42 \mu$ s for amputee subjects, respectively. The Mann-Whitney U-test indicated that the JND of vibration was significantly higher than that of buzz sensation in able-bodied subjects $(\mathrm{p}<0.05)$, but there was no significant difference between them in amputee subjects ( $p>0.05)$. There was no significant difference in the JND of vibration between able-bodied subjects and amputee subjects ( $p>0.05)$, while the buzz sensitivity in amputee subjects was significantly higher than that in able-bodied subjects $(\mathrm{p}<0.05)$, which indicated that these amputee subjects were more sensitive to buzz sensation than able-bodied subjects.

\section{E. Finger-specific identification}

To demonstrate the utility of sensory coding protocol for restoration of natural sensory feedback in forearm amputees, buzz sensation was chosen to encode the pressure from the prosthetic fingertip for its wide range of pulse width modulation at $50 \mathrm{~Hz}$ and low JND in amputees. Results of finger-specific identification experiments were displayed in Fig. 7. The mean accuracy for all amputee subjects was $77.85 \pm 8.15 \%$, but the

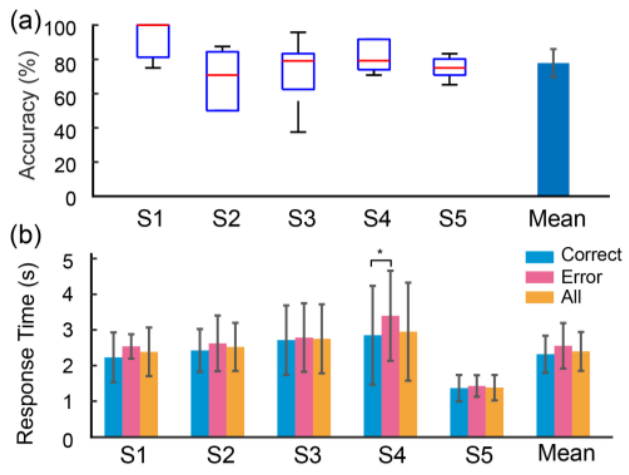

Fig. 7. Results of the finger-specific identification experiment. (a) presents the accuracy of five fingers in amputee subjects (S1-S5) an the mean accuracy (mean $\pm \mathrm{SD}$ ) of all five amputees. (b) shows the response times with correct, wrong identification, and all identifications and the mean response times (mean $\pm \mathrm{SD})$ for all five subjects. Statistical significance is indicated with $*(p<0.05)$.

median accuracy was $100 \%$ for $\mathrm{S} 1,70.8 \%$ for $\mathrm{S} 2,79.1 \%$ for $\mathrm{S} 3$, $79.2 \%$ for S4, and $75.9 \%$ for S5, respectively, which was all above chance probability (20\%) (Fig. 7(a)). Response time to identify single-finger stimulation (Fig. 7(b)) was $2.41 \pm 0.69 \mathrm{~s}$, $2.55 \pm 0.68 \mathrm{~s}, 2.78 \pm 0.98 \mathrm{~s}, 2.98 \pm 1.39 \mathrm{~s}, 1.40 \pm 0.36 \mathrm{~s}$ for S1, S2, S3, S4, and S5, respectively. Note that the average response time for correct identifications was not significantly different from that of wrong identifications across all amputee subjects, except for $\mathrm{S} 4(\mathrm{p}<0.05)$. This implies that incorrect identification may not be caused by cognitive difficulty, but misinformation by the spillover of stimulation current to adjacent PFM areas. The rate of misidentification was low, suggesting that it is feasible to provide finger-specific information to amputee subjects using pulse width modulation of buzz sensation via ETS.

\section{DISCUSSION}

This study evaluated optimal ranges of pulse width modulation of sensations evoked by TENS of PFM regions of forearm amputees with small stimulation electrodes. TENS can elicit referred sensation of discrete digits of lost hand via evoked tactile sensation (ETS) in the PFM regions. This noninvasive neural interface is able to convey near-natural fingerspecific sensory effects by a regenerated direct sensory pathway from peripheral stump skin to the primary somatosensory cortex [23]. In this study, we designed a protocol to determine the pulse width modulation range of sensations by TENS. This protocol may facilitate the process to obtain finger-specific information and encode sensory feedback for hand prostheses. Results confirmed the feasibility to personalize sensory feedback coding strategy for individual forearm amputees.

Sensations evoked by TENS depend on stimulus parameters [28], [29], [36]. Therefore, it is necessary to assess the relationship of the perceptual threshold about amplitude and pulse width for different sensations. Chai et al. [24] found that touch, pressure, vibration, buzz, numb and tingling could be evoked in order with the increase of pulse width, amplitude, or frequency using relatively large surface electrodes with a 25 $\mathrm{mm}$ diameter. Pressure sensation consistently appeared along with other sensations during electrical stimulation. However, in this study, fewer types of sensations were evoked with pulse 
width modulation in the able-bodied and amputee subjects. This may be due to the reduced electrode size here [31]. The evoked sensations were related to stimulus frequency, for example, vibration was more easily induced at lower frequencies, while buzz and numb sensations were likely to be evoked at higher frequencies (Fig. 2). But the sensation of touch and tingling appeared at all stimulation frequencies, which can be recruited more reliably by increasing the intensity of stimuli. Different types of sensations may result from activation of a variety of nerve fibers and cutaneous receptors [25], [26], [37], [38]. Vibration and buzz sensations may arise from activation of rapidly adapting units and Pacinian corpuscles respectively [26], [38].

The perceptual threshold at different amplitudes provided a landscape view of modulation areas for ETS-based sensory feedback. The perceptual threshold displayed a typical inverse relationship between amplitude and pulse width. As amplitude increased, the pulse width required to reach the threshold of specific sensation decreased. At low amplitudes, since some sensations only occurred with long pulse widths that were out of the range of sensory stimulation, it was not able to determine the rheobase of the relationship between amplitude and pulse width. Sensory types varied between subjects (Fig. 3), which may be caused by differences in electrode position, skin condition, and distribution of sensory nerve fibers beneath the skin. Due to limitations in air permeability of adhesive gel of surface electrodes, stimulation electrodes may need to be replaced after a period of time, and sensory modulation range should be recalibrated after replacing electrodes. Nevertheless, the perceptual threshold relationship between amplitude and pulse width provides basic information for the optimal selection of amplitude and frequency that may maximize the modulation ranges of pulse width. Results revealed that vibration and buzz sensations displayed widest modulation ranges of pulse width at $20 \mathrm{~Hz}$ and $50 \mathrm{~Hz}$ respectively (Fig. 3), which was consistent with that at fixed amplitude (Fig. 4 \& Fig. 5). The assessment for perceptual thresholds at different frequencies and amplitudes was a more time-consuming procedure (often more than 10 hours). However, once this was done for a subject, the time to calibrate or recalibrate modulation ranges of pulse width at chosen amplitude and frequency could be significantly reduced to about 30 minutes. This calibration protocol makes it practical to fit sensory feedback to amputees. This feature is valuable to rebuild near-natural sensory feedback in amputees with ETS, or substitutive feedback for amputees without ETS.

Sensation types and perceptual intensities are important ways to convey external information in sensory feedback. In this study, just noticeable difference (JND) was used as a measure to characterize subjects' sensitivity to changes in sensation intensity. The mean JNDs of vibration and buzz sensation in able-bodied and amputee subjects were below $20 \mu$ s, while the modulation ranges for these sensations were about $250 \mu$ s (Fig. $6 \&$ Fig. S2), which suggested that both amputees and ablebodied subjects were able to discriminate multiple levels of intensity for these sensations. Our results showed that the JND almost remained constant across all reference stimuli, as was found in Geng et al [39]. This phenomenon was contrary to Weber's law, in which the JND increased proportionally with the increase of reference stimuli [40]. This irregularity may be due to the non-spatial homogeneity in the recruitment of sensory nerve fibers by TENS. Nevertheless, our results indicate that perceptual intensity can be effectively modulated to convey external information via a specific sensation.

The ultimate goal here is to develop a protocol to encode external information at prosthetic fingers, such as contact pressure, for feedback to amputees. This was demonstrated in the finger-specific identification experiment. Results indicated that amputees could identify contact pressure at prosthetic fingers through ETS with high accuracy. It was interesting to note that S3, who had an irregular response in the JND sensitivity assessment, could still identify the contact finger with high accuracy. The average response time of amputee subjects ranged from 1.40 to $2.98 \mathrm{~s}$ (Fig. 7(b)). Rapid recognition of contact fingers was a requirement for closedloop control of the prosthetic hand. These results suggest that it may be feasible for amputees to use ETS for perception and closed-loop control of prosthetic grasping. In further research, more types of sensations with ETS may be explored to encode other sensory information (such as slipping or nociceptive warning).

There are several limitations in this study. One of them is the lack of measurement of electrode impedance with time, which could result in changes in electrode resistance. Monitoring electrode impedance is useful to stabilize the effects of sensory stimulation in the daily use of the sensitized prosthetic hand. Another limitation of this study is that the upper limit of pulse width modulation was set to $600 \mu$ s due to the possibility to cause discomfort. In practical application, the upper limit of pulse width modulation could be increased appropriately to values without causing discomfort.

\section{v. CONCLUSION}

In this study, modulation ranges of perception with ETS using small-size electrodes are investigated. Results confirm that fewer sensation types are elicited by surface stimulation with smaller electrodes. Evoked sensations also vary with stimulation frequency. The perceptual threshold decreases with increasing current amplitude, which encircles a bounded area in the amplitude/pulse width space for specific sensation. Large modulation areas and high sensitivity for buzz and vibration indicate the possibility to encode a multitude of sensory information with these types of sensations. Sensory effects elicited in amputees are similar to those induced in able-bodied subjects, which suggests that similar sensory encoding protocol can be applied to those amputees without ETS for sensory substitution. Results of finger-specific identification confirm the effectiveness of sensory coding strategy for feedback of prosthetic finger pressure to amputees. This study establishes a general method for customizing the ETS-based, non-invasive sensory feedback from prosthetic hand to human nervous system.

\section{SUPPLEMENTARY MATERIALS}

Fig. S1. Perceptual threshold in pulse width of sensations with variable amplitude in S3, S4, and S5.

Fig. S2. Sensitivity of vibration and buzz sensations in five PFMs of fingers for S1, S4, and S5.

Table S1-S5 The fitted curves and modulation areas of 
sensations for able-bodied subjects (H1-H5), S1, S3, S4, and S5.

\section{REFERENCES}

[1] I. Saunders and S. Vijayakumar, "The role of feed-forward and feedback processes for closed-loop prosthesis control," J. NeuroEng. Rehabil., vol. 8, no. 1, p. 60, Oct. 2011.

[2] S. N. Flesher et al., "Intracortical microstimulation of human somatosensory cortex," Sci. Transl. Med., vol. 8, no. 361, pp. 361ra141361ra141, Oct. 2016.

[3] M. Ortiz-Catalan et al., "Self-contained neuromusculoskeletal arm prostheses," N. Engl. J. Med., vol. 382, no. 18, pp. 1732-1738, Apr. 2020.

[4] M. Markovic et al., "The clinical relevance of advanced artificial feedback in the control of a multi-functional myoelectric prosthesis," $J$. NeuroEng. Rehabil., vol. 15, no. 1, p. 28, Dec. 2018.

[5] E. L. Graczyk et al., "Home use of a neural-connected sensory prosthesis provides the functional and psychosocial experience of having a hand again," Sci. Rep., vol. 8, no. 1, p. 9866, Dec. 2018.

[6] E. D'Anna et al., "A somatotopic bidirectional hand prosthesis with transcutaneous electrical nerve stimulation based sensory feedback," Sci. Rep., vol. 7, no. 1, p. 10930, Dec. 2017.

[7] S. J. Bensmaia et al., "Restoration of sensory information via bionic hands," Nat. Biomed. Eng., Nov. 2020.

[8] M. Armenta Salas et al., "Proprioceptive and cutaneous sensations in humans elicited by intracortical microstimulation," eLife, vol. 7, p. e32904, Apr. 2018.

[9] S. V. Hiremath et al., "Human perception of electrical stimulation on the surface of somatosensory cortex," PLOS ONE, vol. 12, no. 5, p. e0176020, May 2017.

[10] J. A. George et al., "Biomimetic sensory feedback through peripheral nerve stimulation improves dexterous use of a bionic hand," Sci. Robot., vol. 4, no. 32, p. eaax2352, Jul. 2019.

[11] I. Cuberovic et al., "Learning of artificial sensation through long-term home use of a sensory-enabled prosthesis," Front. Neurosci., vol. 13, p. 853, Aug. 2019.

[12] F. M. Petrini et al., "Six-month assessment of a hand prosthesis with intraneural tactile feedback: hand prosthesis," Ann Neurol., vol. 85, no. 1, pp. 137-154, Jan. 2019.

[13] C. Antfolk et al., "Artificial redirection of sensation from prosthetic fingers to the phantom hand map on transradial amputees: vibrotactile versus mechanotactile sensory feedback," IEEE Trans. Neural Syst. Rehabil. Eng., vol. 21, no. 1, pp. 112-120, Jan. 2013.

[14] M. R. Mulvey et al., "The use of transcutaneous electrical nerve stimulation (TENS) to aid perceptual embodiment of prosthetic limbs," Med. Hypotheses, vol. 72, no. 2, pp. 140-142, Feb. 2009.

[15] E. L. Graczyk et al., "Frequency shapes the quality of tactile percepts evoked through electrical stimulation of the nerves," bioRxiv, Aug. 2020.

[16] T. Callier et al., "The frequency of cortical microstimulation shapes artificial touch," Proc. Natl. Acad. Sci. USA, vol. 117, no. 2, pp. 11911200, Jan. 2020

[17] J. L. Segil et al., "Combination of simultaneous artificial sensory percepts to identify prosthetic hand postures: a case study," Sci. Rep., vol. 10 , no. 1 , p. 6576 , Dec. 2020.

[18] S. Wendelken et al., "Restoration of motor control and proprioceptive and cutaneous sensation in humans with prior upper-limb amputation via multiple Utah Slanted Electrode Arrays (USEAs) implanted in residual peripheral arm nerves," J. NeuroEng. Rehabil., vol. 14, no. 1, p. 121 , Dec. 2017.

[19] F. Clemente et al., "Intraneural sensory feedback restores grip force control and motor coordination while using a prosthetic hand," $J$. Neural Eng., vol. 16, no. 2, p. 026034, Apr. 2019.

[20] E. D'Anna et al., "A closed-loop hand prosthesis with simultaneous intraneural tactile and position feedback," Sci. Rob., vol. 4, no. 27, p. $262741,2019$.

[21] J. A. George et al., "Long-term performance of utah slanted electrode arrays and intramuscular electromyographic leads implanted chronically in human arm nerves and muscles," J Neural Eng., vol. 17. no. 5, p. 056042, Apr. 2020.

[22] U. Wijk et al., "Sensory feedback in hand prostheses: a prospective study of everyday use," Front. Neurosci., vol. 14, p. 663, Jul. 2020
[23] M. Hao et al., "Restoring finger-specific sensory feedback for transradial amputees via non-invasive evoked tactile sensation," IEEE Open J. Eng. Med. Biol., vol. 1, pp. 98-107, 2020.

[24] G. Chai et al., "Characterization of evoked tactile sensation in forearm amputees with transcutaneous electrical nerve stimulation," J. Neural Eng., vol. 12, no. 6, p. 066002, Dec. 2015.

[25] A. Y. J. Szeto and F. A. Saunders, "Electrocutaneous stimulation for sensory communication in rehabilitation engineering," IEEE Trans. Biomed. Eng., vol. BME-29, no. 4, pp. 300-308, Apr. 1982.

[26] K. A. Kaczmarek et al., "Electrotactile and vibrotactile displays for sensory substitution systems," IEEE Trans. Biomed. Eng., vol. 38, no. 1, pp. 1-16, Jan. 1991.

[27] E. A. Pfeiffer, "Electrical stimulation of sensory nerves with skin electrodes for research, diagnosis, communication and behavioral conditioning: A survey," Med. \& biol. Engng., vol. 6, no. 6, pp. 637651, Nov. 1968.

[28] M. D. Alonzo et al., "Electro-cutaneous stimulation on the palm elicits referred sensations on intact but not on amputated digits," J. Neural Eng., vol. 15, no. 1, p. 016003 , Feb. 2018.

[29] A. Y. J. Szeto, "Electrocutaneous code pairs for artificial sensory communication systems," Ann Biomed Eng, vol. 10, no. 4, pp. 175-192, Jul. 1982.

[30] A. Y. J. Szeto and J. Lyman, "Comparison of codes for sensory feedback using electrocutaneous tracking," Ann. Biomed. Eng., vol. 5, no. 4, pp. 367-383, Dec. 1977.

[31] P. Li et al., "Effects of electrode size and spacing on sensory modalities in the phantom thumb perception area for the forearm amputees," in Proc. Annu. Int. Conf. IEEE Eng. Med. Biol. Soc., Milan, Aug. 2015, pp. 3383-3386.

[32] T. Wang et al., "Perceptual attributes of cutaneous electrical stimulation to provide sensory information for prosthetic limb," in IEEE Int. Conf. Inf. Sci. Technol., Mar. 2013, pp. 22-25.

[33] J. Ara et al., "Effects of the stimulus parameters on the tactile sensations elicited by single-channel transcutaneous electrical stimulation," in Int. J. Precis. Eng. Manuf. vol. 15, Feb. 2014, pp. 305-313.

[34] T. Tashiro and A. Higashiyama, "The perceptual properties of electrocutaneous stimulation: Sensory quality, subjective intensity, and intensity-duration relation," Perception \& Psychophysics, vol. 30, no. 6, pp. 579-586, Nov. 1981

[35] F. Yang et al., "An experimental protocol for evaluating pulse width modulation ranges of evoked tactile sensory feedback in amputees," in Proc. Annu. Int. Conf. IEEE Eng. Med. Biol., Jul. 2020, pp. 3869-3872.

[36] B. Geng et al., "Evaluation of sensation evoked by electrocutaneous stimulation on forearm in nondisabled subjects," JRRD, vol. 49, no. 2, p. 297, 2012.

[37] R. Butikofer and P. D. Lawrence, "Electrocutaneous nerve stimulationi: model and experiment," IEEE Trans. Biomed. Eng., vol. BME-25, no. 6, pp. 526-531, Nov. 1978.

[38] J. L. Ochoa and H. E. Torebjörk, "Sensations evoked by intraneural microstimulation of single mechanoreceptor units innervating the human hand," J. Physiol., vol. 342, pp. 633-654, 1983.

[39] B. Geng et al., "Psychophysical evaluation of subdermal electrical stimulation in relation to prosthesis sensory feedback," IEEE Trans. Neural Syst. Rehabil. Eng., vol. 26, no. 3, pp. 709-715, Mar. 2018.

[40] G. A. Gescheider, Psychophysics: the fundamentals, 3rd ed. Mahwah, N.J: L. Erlbaum Associates, 1997. 